\title{
Negative Pressure Wound Therapy of Chronically Infected Wounds Using 1\% Acetic Acid Irrigation
}

\author{
Hii Sun Jeong ${ }^{1}$, Byeong Ho Lee ${ }^{1}$, Hye Kyung Lee ${ }^{2}$, Hyoung Suk Kim³ ${ }^{3}$, Min Seon Moon ${ }^{3}$, \\ In Suck Suh ${ }^{1}$ \\ ${ }^{1}$ Department of Plastic and Reconstructive Surgery, Kangnam Sacred Heart Hospital, Hallym University College of Medicine, Seoul; \\ ${ }^{2}$ Department of Plastic and Reconstructive Surgery, Eulji General Hospital, Eulji University College of Medicine, Seoul; ${ }^{3}$ Department of Plastic \\ and Reconstructive Surgery, Myongji Hospital, Kwandong University College of Medicine, Goyang, Korea
}

Background Negative-pressure wound therapy (NPWT) induces angiogenesis and collagen synthesis to promote tissue healing. Although acetic acid soaks normalize alkali wound conditions to raise tissue oxygen saturation and deconstruct the biofilms of chronic wounds, frequent dressing changes are required.

Methods Combined use of NPWT and acetic acid irrigation was assessed in the treatment of chronic wounds, instilling acetic acid solution (1\%) beneath polyurethane membranes twice daily for three weeks under continuous pressure $(125 \mathrm{~mm} \mathrm{Hg})$. Clinical photographs, $\mathrm{pH}$ levels, cultures, and debrided fragments of wounds were obtained pre- and posttreatment. Tissue immunostaining (CD31, Ki-67, and CD45) and reverse transcription-polymerase chain reaction (vascular endothelial growth factor [VEGF], vascular endothelial growth factor receptor [VEGFR]; procollagen; hypoxia-inducible factor 1 alpha [HIF-1-alpha]; matrix metalloproteinase [MMP]1,-3,-9; and tissue inhibitor of metalloproteinase [TIMP]) were also performed.

Results Wound sizes tended to diminish with the combined therapy, accompanied by drops in wound $\mathrm{pH}$ (weakly acidic or neutral) and less evidence of infection. CD31 and Ki-67 immunostaining increased $(\mathrm{P}<0.05)$ post-treatment, as did the levels of VEGFR, procollagen, and MMP-1 $(P<0.05)$, whereas the VEGF, HIF-1-alpha, and MMP-9/TIMP levels declined $(\mathrm{P}<0.05)$.

Conclusions By combining acetic acid irrigation with negative-pressure dressings, both the $\mathrm{pH}$ and the size of chronic wounds can be reduced and infections be controlled. This approach may enhance angiogenesis and collagen synthesis in wounds, restoring the extracellular matrix.

Keywords Acetic acid / Biofilm / Negative pressure wound therapy
Correspondence: Hii Sun Jeong Department of Plastic and Reconstructive Surgery, Kangnam Sacred Heart Hospital, Hallym University Medical Center, Hallym University College of Medicine, 1 Singil-ro, Yeongdeungpo-gu, Seoul 150-950, Korea

Tel: +82-2-829-5182

Fax: +82-2-847-5183

E-mail: hiisunj@gmail.com
This article was presented at 72nd Congress of The Korean Society of Plastic and Reconstructive Surgeons on November 7-9, 2014 in Seoul, Korea.

This work was supported by the Myongji Hospital Research Fund.

No potential conflict of interest relevant to this article was reported.

\section{INTRODUCTION}

Chronic wounds, which typically result from medical condi- tions, nutritional deficiencies, infections, and metabolic disorders, involve intensive and time-consuming treatments. Although recent medical advances have prolonged life, the diffi- 
cult-to-heal wounds (i.e., sores and dia betic feet) that accompany chronic disease states have increased in incidence, commensurate with the expanding elderly population. Such wounds are often infected by highly virulent bacterial organisms, namely methicillin-resistant Staphylococcus aureus (MRSA) and Pseudomonas aeruginosa, that colonize biofilms and complicate treatment $[1,2]$.

Negative-pressure wound therapy (NPWT), which deters the accumulation of fluid at wound sites through continuous drainage, makes daily dressing changes unnecessary, improves regional blood flow, and reduces bacterial proliferation, thus limiting the opportunity for infection. At the cellular level, NPWT is also known to encourage collagen synthesis, angiogenesis, and granulation tissue formation [3-5]. Unfortunately, NPWT with polyurethane foam has the limitation that it is mainly used for wounds with controlled infection after procedures such as necrotic tissue debridement.

Acetic acid, on the other hand, creates weakly acidic or neutral states in wounds that boost oxygen saturation, and healing is promoted by the degradation of surface biofilms [6-9]. Because acetic acid soaks require at least two dressing changes daily, negative-pressure dressings seemed an ideal pairing, facilitating acid instillation by design. Therefore, we assessed the clinical benefit of combining both elements in several test patients with recalcitrant chronic sores.

\section{METHODS}

\section{Patients}

Three patients were studied, all of whom suffered treatment-refractory ( $>3$ weeks), non-healing, and bacteria-laden wounds between September 1, 2011 and June 30, 2012. The following parameters were grounds for exclusion: 1) malignant tumors, 2 ) systemic inflammatory diseases, 3) medications that might delay wound healing (i.e., steroids, immunosuppressants, or chemotherapy), 4) uncontrolled diabetes (blood glucose $>200 \mathrm{mg} /$ dL), 5) nutritional deficiencies, and 6) poor vital signs.

\section{Methods}

\section{Wound dressing procedure}

Eight holes ( $3 \mathrm{~mm}$ across) were made in the last $10 \mathrm{~cm}$ of conventional intravenous lines, which were circularly placed atop wounds. Ports for acetic acid injection were made outside the vacuum dressings and were maintained aseptically (Fig. 1). For a period of three weeks, vacuum-assisted closure (Kinetics Concepts Inc., San Antonio, Texas, USA) dressings were routinely applied under continuous pressure $(125 \mathrm{~mm} \mathrm{Hg}$ ) and changed twice weekly. NPWT and compressive dressings were used ex-

\section{Fig. 1. Use of NPWT and acetic acid irrigation}

A port of acetic acid injection was secured aseptically outside a negative-pressure dressing. Conventional intravenous lines were placed under the vacuum dressing. NPWT, negative-pressure wound therapy.

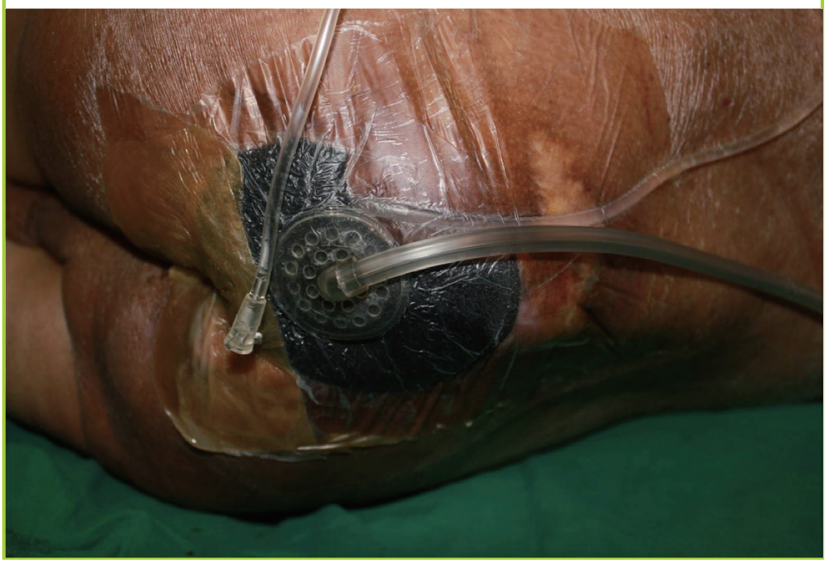

clusively during the treatment phase, irrigating wounds twice daily with $30-\mathrm{mL}$ aliquots of $1 \%$ acetic acid solution. Because these patients had severe wound infection, we performed full debridement, including removing a little healthy tissue in the first stage, and performed debridement using curettage twice a week during the three weeks of NPWT. Wound size and $\mathrm{pH}$ were determined pre- and post-treatment. The debrided tissue was used for histological assessment and quantitative real-time polymerase chain reaction (RT-PCR) studies.

\section{Wound size, $\mathrm{pH}$, and culture protocols}

The wound size (with photographs) was measured at the baseline and then weekly (weeks 1-3) during the combination of NPWT and acetic acid irrigation. Two-dimensional wound sizes were recorded. Wound bursae were then identified (drawn with markers on aseptic films) and reproduced for scanning at constant resolution ( $300 \mathrm{dpi})$, with areas calculated using the ImageJ software (National Institutes of Health-Scion Co., Bethesda, MD, USA).

Wound $\mathrm{pH}$ was determined using color-coded litmus test paper (Advantec, Toyo Roshi Kaisha Ltd., Tokyo, Japan) weekly. Three physicians confirmed each test result.

Tissue culture was performed at initial wound debridement and 1, 2, and 3 weeks after combined NPWT/acetic acid irrigation. Microbial proliferation was gauged as much, some, little, or no growth.

\section{Histological examination}

Histological studies were conducted before and after therapy, using 3-mm rims of tissue obtained from the wound margins at debridement for routine hematoxylin and eosin (H\&E) setions 
(as general reference) and immunohistochemical staining of CD31 (ab28364, Abcam, Cambridge, MA, USA), CD45 (ab10558, Abcam), and Ki-67 (ab15580, Abcam) molecular markers. All samples were formalin fixed and paraffin embedded. Standard indirect immunoperoxidase procedures (avidinbiotin-peroxidase complex) were followed for the various immunostains. The Ki-67 marker was targeted first. After blocking any antibody to $\mathrm{CD} 31$ (1:20 dilution), sections were incubated at $4^{\circ} \mathrm{C}$ overnight. CD31-positive cells were identified by red cytoplasmic staining, and brown nuclear staining indicated cells positive for Ki-67. To gauge angiogenesis, the capillary density/ high-power field was calculated from five fields in CD31-immunostained sections, using medians of three values (maximum and minimum values excluded). Cellular proliferation was determined using Ki-67 and CD45 markers. Photomicrographs $(200 \times)$ of each Ki-67-immunostained slide were obtained at five fields, using imaging analysis software (MetaMorph, Universal Imaging Co., Downingtown, PA, USA) to quantify the stained cells. Medians were generated from three values, excluding maximum and minimum values $[10]$.

\section{Quantitative real-time polymerase chain reaction}

Two specimens each were harvested during the initial and the last debridement. A specimen was divided into five samples. The total RNA was extracted from cells cultured in a monophasic solution of phenol and guanidine isothiocyanate (TRIzol reagent, Life Technologies-Invitrogen, Milan, Italy). In brief, the cells were lysed by adding $1.0 \mathrm{~mL}$ of an extraction reagent, and the total RNA was isolated according to the manufacturer's instructions. Complementary DNA was synthesized from $400 \mathrm{ng}$ of total RNA per sample through 12 -cycle $\left(30\right.$ seconds at $37^{\circ} \mathrm{C}$, 4 minutes at $48^{\circ} \mathrm{C}$, and 30 seconds at $\left.55^{\circ} \mathrm{C}\right)$ and 1 -cycle $(10$ minutes at $95^{\circ} \mathrm{C}$ ) incubations by using Moloney murine leukemia virus reverse transcriptase (AccuPower RocketScript Cycle RT PreMix, Bioneer Co., Daejeon, Korea) and oligo (dT) priming. Amplification was performed in a programmable thermal controller (MyGenie 96 Gradient Thermal Block, Bioneer Co.) by using recombinant Taq DNA polymerase (AccuPowe GreenStar PCR PreMix, Bioneer Co.). Parallel amplification of cDNA for the housekeeping enzyme, glyceraldehyde-3-phosphate dehydrogenase (GAPDH), served as the internal control. To enable semiquantitative between-sample comparisons, serial three-fold dilutions of cDNA (corresponding to 100-1.2 ng of the total) were subjected to increasing PCR cycles (2340 ), thus defining the linear amplification range of each primer set. All cDNA samples were then amplified at cycle numbers optimal for the genes of interest. This was 40 cycles ( 5 seconds at $95^{\circ} \mathrm{C}, 25$ seconds at $58^{\circ} \mathrm{C}$, and 30 seconds at $72^{\circ} \mathrm{C}$ ) for vas- cular endothelial growth factor A (VEGFA), vascular endothelial growth factor receptor (VEGFR), procollagen, hypoxia-inducible factor 1 alpha (HIF-1- $\alpha$ ), matrix metalloproteinase 1 (MMP-1), matrix metalloproteinase 3 (MMP-3), matrix metalloproteinase 9 (MMP-9), and tissue inhibitor of metalloproteinase (TIMP) and 24 cycles ( 5 seconds at $95^{\circ} \mathrm{C}, 25$ seconds at $58^{\circ} \mathrm{C}$, and 30 seconds at $72^{\circ} \mathrm{C}$ ) for procollagen and GAPDH, each preceded by a 10 -min denaturation step at $95^{\circ} \mathrm{C}$ and followed by a 2 -minute elongation step at $65^{\circ} \mathrm{C}$. Results were expressed as percentages of signals obtained from the parallel amplification of GAPDH in the same RT product. To quantify bands, ImageJ software (National Institutes of Health-Scion) was used [11-14].

\section{Ethical considerations}

All research involving human participants was approved by the Institutional Review Board of a single facility (IRB No.11-010), and all clinical investigations were aligned with the Declaration of Helsinki principles. Prior to the analysis, all patient data remained anonymous. Written approval to use the accompanying photographs in research, presentations, and publications was provided by the patients.

\section{Statistics}

The Wilcoxon signed-rank test and paired T-test were used for our purposes. Both methods are used to compare matched samples, such as change from one time point to another or exposure to more than one condition. The analysis relied on standard software (IBM SPSS ver. 21, IBM Co., Armonk, NY, USA).

\section{RESULTS}

\section{Wound size, $\mathrm{pH}$, and culture}

All three patients had sacral sores, ranging in duration from 6 to 24 months. Wound sizes of $24 \mathrm{~cm}^{2}, 15 \mathrm{~cm}^{2}$, and $8 \mathrm{~cm}^{2}$ at baseline dropped to $13.5 \mathrm{~cm}^{2}$ (44\% lower) (Fig. 2), $12 \mathrm{~cm}^{2}$ (25\% lower) (Fig. 3), and $6 \mathrm{~cm}^{2}$ (25\% lower) (Fig. 4), respectively. Bursa sizes also declined 30\% $\left(61.75 \rightarrow 43.75 \mathrm{~cm}^{2}\right), 33 \%(30 \rightarrow$ $\left.20 \mathrm{~cm}^{2}\right)$, and $20 \%\left(20 \rightarrow 16 \mathrm{~cm}^{2}\right)$, respectively (Table 1$)$.

In all three wounds, $\mathrm{pH}$ levels that were strongly alkaline at baseline $(9.0,10.0$, and 8.0 ) became weakly alkaline or neutral (8.0, 8.0, and 7.0, respectively) after treatment. Proteus mirabilis, Escherichia coli, Streptococcus, MRSA, and Candida albicans were variably isolated from each of the three wounds. Over time, colonization either declined or cleared (no growth) (Table 1).

\section{Histological examination}

H\&E-stained tissue sections showed increased collagen deposi- 


\section{Fig. 2. Patient 1}

Photographs at (A) baseline and (B) after the three-week treatment period.

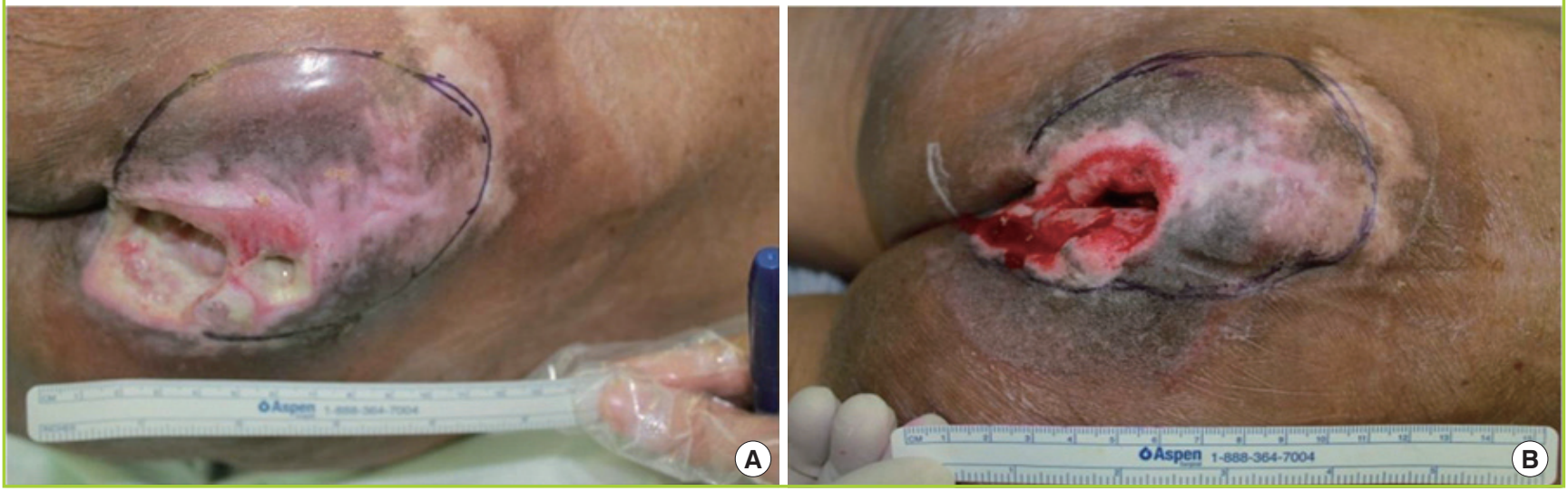

Fig. 3. Patient 2

Photographs at (A) baseline and (B) after the three-week treatment period.

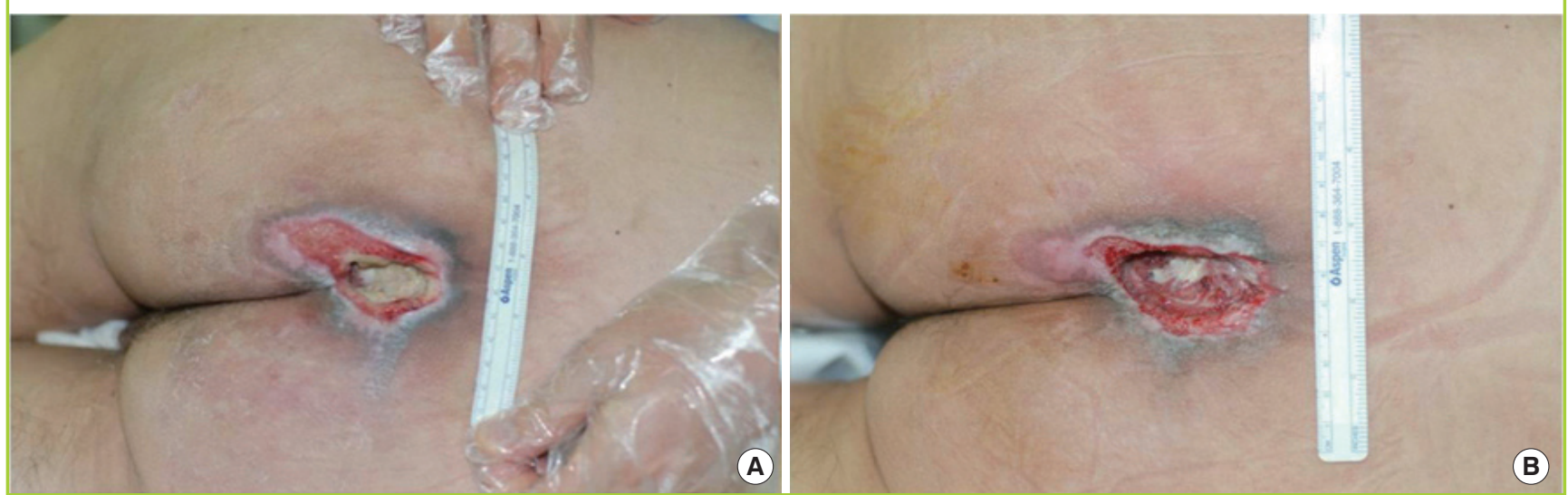

Fig. 4. Patient 3

Photographs at (A) baseline, (B) after initial debridement, and (C) following the three-week treatment.
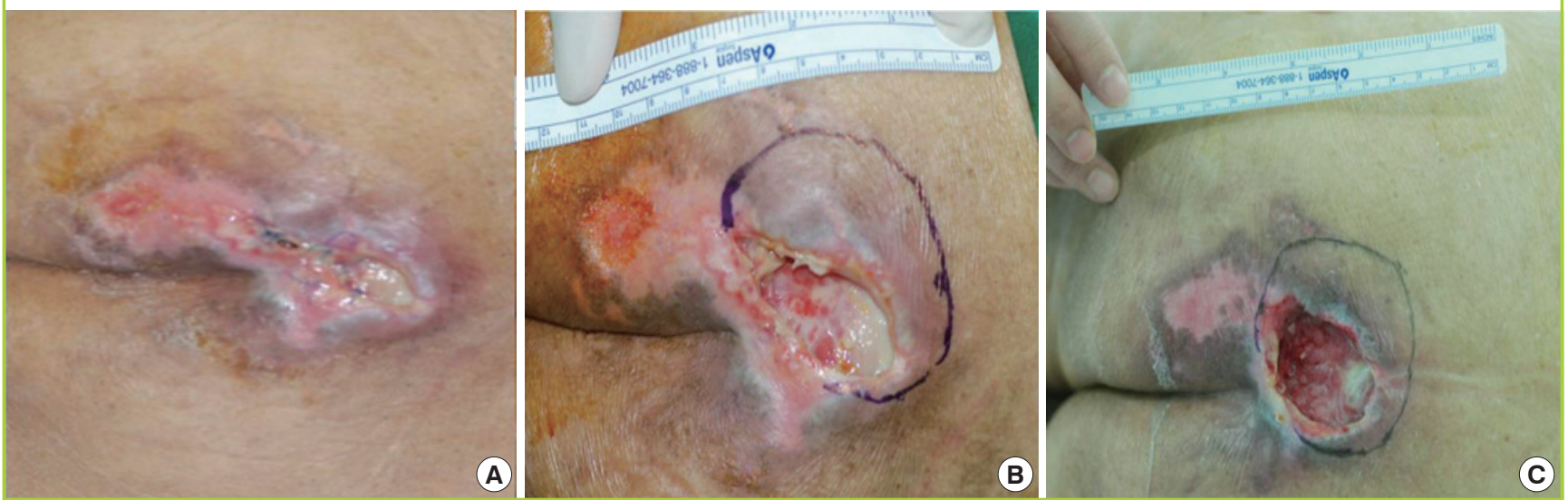

tion $(40 \times$ and $100 \times$ views $)$. Post-treatment immunostaining of CD31 $(\mathrm{P}=0.035)$ and $\mathrm{Ki}-67(\mathrm{P}=0.028)$ markers increased significantly (Figs. 5, 6), although slight reduction or no change in immunostaining of CD45 $(\mathrm{P}=0.142)$ was observed (Wil- coxon signed-rank test).

Quantitative real-time polymerase chain reaction In the aftermath of the treatment, the expression of VEGFA 
Table 1. Comparison of wound area, bursa, pH, and wound culture

\begin{tabular}{|c|c|c|c|c|c|c|c|c|c|c|c|c|c|c|c|c|}
\hline \multirow{2}{*}{$\begin{array}{l}\text { Case } \\
\text { no. }\end{array}$} & \multicolumn{4}{|c|}{ Wound area $\left(\mathrm{cm}^{2}\right)$} & \multicolumn{4}{|c|}{ Bursa $\left(\mathrm{cm}^{2}\right)$} & \multicolumn{4}{|c|}{$\mathrm{pH}$} & \multicolumn{4}{|c|}{ Culture } \\
\hline & $0 \mathrm{wk}$ & $1 \mathrm{wk}$ & $2 \mathrm{wk}$ & 3 wk & $0 \mathrm{wk}$ & $1 \mathrm{wk}$ & $2 w k$ & $3 \mathrm{wk}$ & $0 \mathrm{wk}$ & $1 \mathrm{wk}$ & $2 \mathrm{wk}$ & $3 \mathrm{wk}$ & $0 \mathrm{wk}$ & $1 \mathrm{wk}$ & $2 \mathrm{wk}$ & $3 \mathrm{wk}$ \\
\hline 1 & 24 & 23 & 17.5 & 13.5 & 61.75 & 53.5 & 51 & 43.75 & 9.0 & 7.0 & 8.0 & 8.0 & $\begin{array}{c}\text { Proteus mirabilis, } \\
\text { Escherichia coli } \\
\text { (many) }\end{array}$ & $\begin{array}{c}\text { Proteus mirabilis, } \\
\text { Escherichia coli } \\
\text { (few) }\end{array}$ & $\begin{array}{c}\text { Proteus mirabilis, } \\
\text { Escherichia coli } \\
\text { (some) }\end{array}$ & MRSA (few) \\
\hline 2 & 16 & 15 & 12.5 & 12 & 30 & 28.5 & 22 & 20 & 10.0 & 9.0 & 8.0 & 8.0 & MRSA (many) & MRSA (many) & MRSA (few) & MRSA (few) \\
\hline 3 & 8 & 7 & 7 & 6 & 20 & 19 & 17 & 16 & 8.0 & 7.5 & 8.0 & 7.0 & $\begin{array}{c}\text { Candida albicans } \\
\text { (few) }\end{array}$ & $\begin{array}{c}\text { Streptococcus } \\
\text { (few) }\end{array}$ & No growth & No growth \\
\hline
\end{tabular}

MRSA, namely methicillin-resistant Staphylococcus aureus.

\section{Fig. 5. Comparison of vascular proliferation (immunostaining of CD31)}

Vascular ingrowth $(200 \times)$ at baseline $(A)$ and after the three-week treatment period (B) visibly increased, denoted with black arrow at the vessel. Immunostaining of CD31 (C) significantly enhanced post-treatment (sample size of each group, $n=15 ;{ }^{\text {a) }} P<0.05$, Wilcoxon signed-rank test). Pre, pre-treatment; Post, post-treatment.
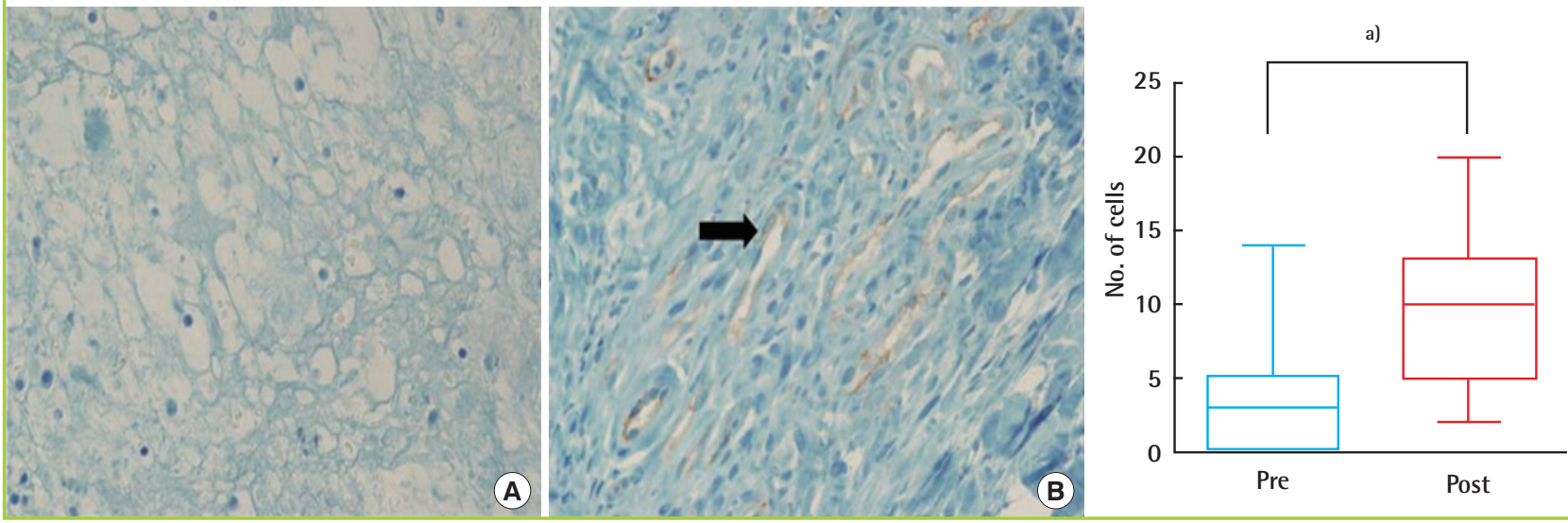

Pre

Post

\section{Fig. 6. Comparison of collagen synthesis (immunostaining of Ki-67)}

Collagen synthesis $(200 \times)$ at baseline $(A)$ and after the three-week treatment period (B); abundance of collagen is shown by black arrow. Immunostaining of Ki-67 (C) significantly enhanced post-treatment (sample size of each group, $n=15$; ${ }^{\text {al }} \mathrm{P}<0.05$, Wilcoxon signed-rank test).
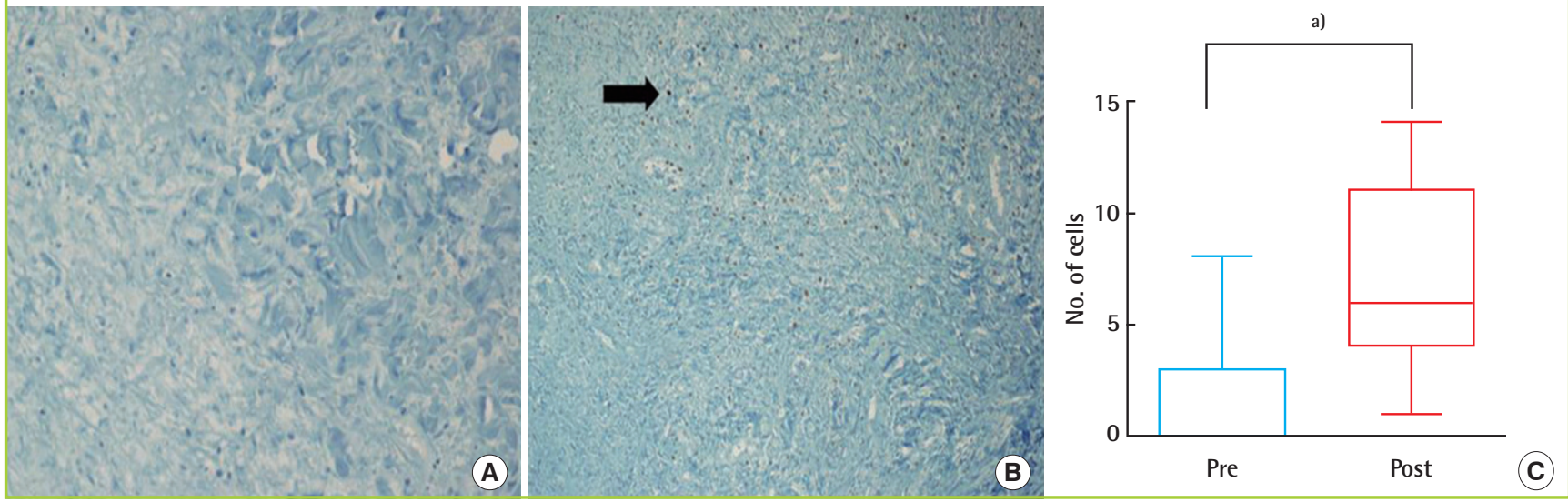

mRNA decreased significantly $(\mathrm{P}<0.001)$ and that of VEGFR mRNA increased significantly $(\mathrm{P}<0.001)$, as did procollagen synthesis $(\mathrm{P}<0.001)$ and MMP-1 levels $(\mathrm{P}<0.001)$, whereas HIF-1-alpha levels declined $(\mathrm{P}<0.001)$. MMP-3 levels did not differ significantly, but MMP-9/TIMP ratio ratios declined sig- nificantly $(\mathrm{P}=0.016)$ after therapy (paired t-test) (Fig. 7).

\section{Complications}

No complications, such as bleeding or hematoma, were encountered during the three-week course of NPWT. Infection was 


\section{Fig. 7. Quantitative RT-PCR matched-sample comparisons}

VEGFA, VEGFR, procollagen, HIF1- $\alpha$, MMP-1, MMP-3, and MMP-9/ TIMP (sample size of each group, $n=30,{ }^{\text {a) }} \mathrm{P}<0.001$; NS, $\mathrm{P}>0.05$; paired T-test). RT-PCR, real-time polymerase chain reaction; VEGFA, vascular endothelial growth factor $A$; VEGFR, vascular endothelial growth factor receptor; HIF1- $\alpha$, hypoxia-inducible factor 1 alpha; MMP-1, matrix metalloproteinase 1; MMP-3, matrix metalloproteinase 3; MMP-9/TIMP, matrix metalloproteinase 9/tissue inhibitor of metalloproteinases; Pre, pre-treatment; Post, post-treatment.

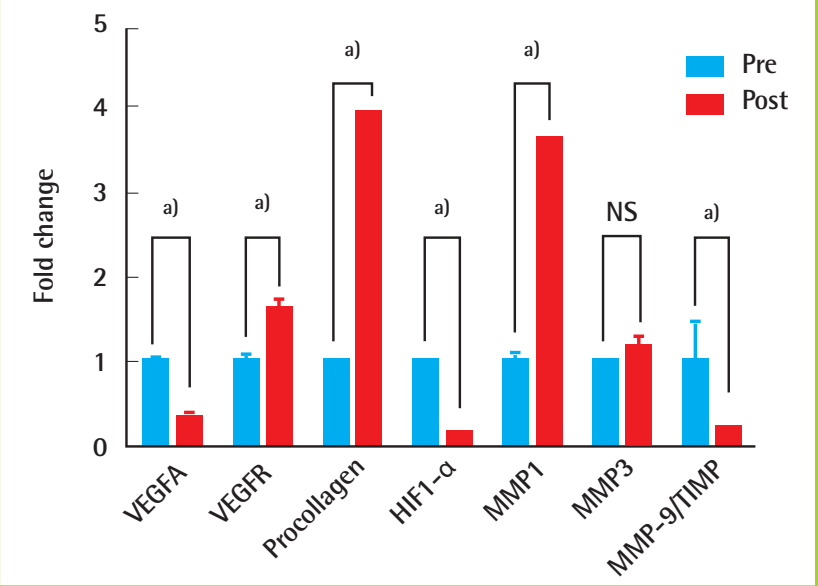

controlled, enabling flap surgery and discharge in each instance, without postoperative infection or wound dehiscence.

\section{DISCUSSION}

Inflammation, proliferation, and maturation are distinct phases in the progression of wound healing, displaying a regression curve over time with a graded effect [15]. Wounds are further categorized as acute, chronic, or chronic nonhealing, the last of these marked by $>6$ months of conventional routine wound care. Conditions favoring delayed wound healing include advanced age, diabetes mellitus (DM), sustained pressure, vascular insufficiency, renal failure, jaundice, infection, and immunosuppression. In older patients, a multiplicity of these conditions is apt to be at play. Conservative treatment may be the only recourse for patients taking prohibitive medication (such as aspirin) or whose overall status is unacceptable for surgical management.

In the case of large wounds, infections are difficult to control, and therefore, the wounds may themselves contribute substantially to the general deterioration of the already weakened patients. Although conservative treatment with antibiotics and dressings may control infections, the emergence of multidrugresistant bacterial strains has limited this approach. In some circumstances, patients may only be candidates for dressing changes in isolation, with no chances of debridement and reconstructive surgery. This further impacts in-hospital infection rates and durations of hospital stay, adding to the burden of healthcare.
Normal skin pH (range, 4-6) is weakly acidic, due to the amino and lactic acid contents of the horny layer and cutaneous adnexa. The acidity of skin contributes to its barrier function [16]. In chronic open wounds, a weakly alkaline $\mathrm{pH}$ range of 7.15-8.9 (8.0 on average) encourages the growth of ammonia-producing and $\mathrm{CO}_{2}$-generating bacteria [16-18]. Ammonia is directly toxic to wounds, inflicting injury through heightened tissue protease activity. Tissue ischemia then ensues, marked by elevated saturated oxyhemoglobin and diminished oxygen partial pressure. Greater acidity helps to reverse these effects [18]. Ultimately, an acid environment is destructive to bacteria and normalizes the wound milieu. $\mathrm{NH}_{3}{ }^{-}$is reduced to $\mathrm{NH}_{4}$, thus restoring normal protease activity and encouraging healing [17].

The importance of skin $\mathrm{pH}$ was first underscored by the addition of acetic acid to a honey-based remedy (Medihoney, Medihoney Ltd., Queensland, Austria) approved in Europe for the treatment of chronic wounds (venous ulcers, DM foot, and burns) [2]. Dressings soaked in 5\% dilute acetic acid and applied to wounds with pseudomonas infections ( $P$. aeruginosa) produced positive clinical results in case studies during the 1960s [6]. However, the short product half-life $(<1 \mathrm{hr})$, necessitating frequent dressing changes, was a clear disadvantage. Ryssel et al. [7-9] studied the in vitro sensitivity of pseudomonas strains commonly encountered in burn centers, confirming the strong antimicrobial effects of acetic acid on P. vulgaris, P. aeroginosa, and A. baumanii-organisms often present in persistent wounds, despite long-term antibiotic therapy [7-9]. MRSA proved sensitive as well.

The high viscosity of honey is also beneficial, eliminating any exudates present and otherwise creating a damp environment optimal for wound healing. Keratinocyte and fibrocyte survival rates are enhanced under such conditions. Honey also contains glucose oxidase, which adds acidity through the conversion of glucose to gluconic acid (measured $\mathrm{pH}=3.5$ ). Acidity alters biofilms that block the delivery of drugs to chronic wounds and exerts antibacterial effects. Aided by bacterial polysaccharide and exudates, biofilms adhere to wound surfaces, are difficult to remove, and serve as reservoirs for continuous microbial release [2]. According to recent reports, a majority (65\%) of infectious diseases are attributable to biofilms [19].

It is clear that acetic acid promotes wound healing, enzymatic activity, and immunity, and attenuates microbial proliferation and biofilm virulence [18]. However, olfactory stimulation (at least initially) and the need for frequent dressing changes are notable drawbacks. At a higher acidic concentration (for greater antibiotic effect), patients will experience more pain [7].

Nonoperative NPWT of the existing complex defects is now widely used, owing to national medical insurance coverage 
(2010) and miniaturization of equipment. The vacuum-assisted closure device (Kinetics Concepts Inc.), consisting of a porous polyurethane sponge (applied to wounds) for moisture and a vacuum-connected film dressing, was first introduced in 1997 under negative pressure, exudates are extracted, the blood supply is increased, and fewer dressing changes are required [3, 5]. As explained by Scherer et al. [5], negative pressure boosts collagen synthesis and neovascularization by wound transformation (macrodeformation) and three-dimensional migration (microdeformation), both encouraging DNA synthesis [5]. In 2011, de Laat et al. [3] found that wound healing was two times faster with NPWT (vs. sodium hypochlorite) in a prospective randomized study, and Kim et al. [4] reported a 95\% rate of limb salvage with NPWT of the DM foot.

In according to international guide line on NPWT with instillation, debridement of biofilms and necrotic tissues is essentially needed and provide to favorable $\mathrm{pH}$ milieu for wound healing $[20,21]$. In addition, once weekly debridement tends to restart the wound-healing cycle, interrupting fibroblast senescence at the inflammatory stage [15]. However, patients who have DM feet, pressure sores, and venous ulcers and who are taking anticoagulants or lack ready access to facilities may forego this step. Consequently, the outcomes of NPWT may fall short of expectations. Furthermore, most of these patients harbor serious hospital-acquired infectious organisms, such as MRSA, vancomycin-resistant Enterococcus, and carbapenemresistant gram-negative bacilli (A. baumani, $P$. areuginosa, and $K$. pneumoniae). In 2009, Arias and Murray [1] reported on multidrug resistant strains and the use of antibiotics in the 21st century, emphasizing that antibiotic choices for these strains are few and carry toxicities.

Our approach combines the convenience of NPWT with the infection control of acetic acid irrigation. The three patients that we treated had infected wounds, with excessive exudation, local redness, warmth, poor granulation tissue, and foul odor. Given the sacral locations, fecal contamination was likely and proved difficult to manage. The wound sites failed to improve, with infectious symptoms persisting, despite three weeks of conventional wound care. Although film dressings are essential to avoid fecal contamination, the increased $\mathrm{CO}_{2}$ of a closed system is problematic, which is why vacuum dressings are vulnerable to infection. Infection control is mandatory for wounds of this nature in order to ensure that healing (i.e., granulation tissue and surface vascularization) takes place.

NPWT and instillation have been combined in many earlier attempts, but guidelines for the same have been set recently [20]. We used twice daily $1 \%$ acetic acid irrigation, delivered in $30-\mathrm{mL}$ aliquots over a period of 15-30 minutes and it differs little from the guideline. A reduction in wound $\mathrm{pH}$ resulted (strongly alkaline at baseline; weakly alkaline or neutral posttreatment) [22,23]. In addition, all pathogenic bacteria identified in wound cultures (MRSA, P. mirabilis, C. albicans) dissipated or cleared. Unlike antibiotic therapy, this approach effectively controlled infection, irrespective of the culpable organisms.

In chronic wounds, MMP-9/TIMP ratios appear to reflect the actual proteolytic environments, more so than either determinant does alone. The significant decline in the MMP-9/ TIMP ratio (based on the RT-PCR assay) seen in our patients post-treatment suggests abatement of prolonged inflammation and wound chronicity $[14,24,25]$. Indeed, the odor grew less intense on physical exams and the exudates retracted visibly, indicating that infection control is clearly fundamental to wound healing.

Re-epithelialization (from the edges of soft tissue defects) and angiogenesis are enabled by the migration of fibroblasts along fibrin networks. Factors promoting inflammation (IL-1a, IL$1 b$, IL-6, and tumor necrosis factor a), facilitating collagen synthesis (fibroblast growth factor [FGF]-2, insulin-like growth factor- 1 , and transferring growth factor $\mathrm{b}$ [TGF- $\mathrm{b}]$ ), converting fibroblasts to myofibroblasts (TGF-b), and initiating angiogenesis (EGF, FGF-2, and TGF-a), are released by platelets and white blood cells at the wound sites. The latter also aid in reepithelialization. In our patients, significant post-treatment increases in CD31 staining and VEGFR expression (vs. baseline levels) corresponded with enhanced tissue vascularization. Ki67 staining and procollagen levels also increased significantly, reflecting the active proliferation of fibroblasts and collagen synthesis. Proliferation and migration of keratinocytes at wound sites (for re-epithelialization) were similarly boosted, as indicated by a significant increase in MMP-1 after therapy. Finally, HIF-1-alpha (a gauge of tissue hypoxia) significantly declined following treatment.

At wound margins, cells must loosen cell-to-cell and cell-toextracellular matrix contacts in order to migrate and re-epithelialize wound surfaces. Multiple MMPs have been implicated from this aspect of wound repair, including MMP-1, -3, -7, -9, $-10,-14$, and -28 . MMP-1 (collagenase- 1 ) is present in human skin wounds during re-epithelialization but diminishes once closure is complete. The expression of MMP- 1 is stimulated by the binding of integrin to type-I collagen (abundant in the dermis). MMP-3 (stromelysin-1) is concentrated in cells behind the migrating front [11]. In our test patients, a statistically significant post-treatment increase in MMP-1 (vs. baseline) signaled that the process of re-epithelialization was well underway [11]. Moreover, reductions in surface areas (average, 35\%) and 
capsule sizes (average, 29\%) of wounds were documented.

Our experiment used highly infected wounds (high risk) to which NPWT with just black polyurethane ether foam, GranuFoam (Kinetics Concepts Inc., San Antonio, Texas, USA) cannot be applied in the first place. Further, we believe that a combination of NPWT and acetic acid irrigation can change the infected wound from high risk to low risk and help enhance collagen synthesis, angiogenesis, and inflammation control, and have proved it from the perspective of molecular biology. Moreover, we gathered tissue specimens that were used in immunohistochemistry and quantitative polymerase chain reaction instead of the collected fluid.

From our perspective, combining a vacuum dressing with acetic acid irrigation is beneficial in many ways. Although film dressings help prevent fecal contamination (particularly in pressure sores), extensive wound irrigation and debridement should be carried out anyway with each dressing change. Acidic wound irrigation unquestionably reduces the bacterial burden, and when combined with NPWT, the protease-induced inflammatory phase of wounds is stabilized, lowering the MMP-9/TIMP ratio. Similarly, the acidification of alkaline wounds reduces ammonia toxicity, creating a more favorable wound milieu (i.e., reduced saturation of oxyhemoglobin and increased partial pressure of oxygen).

Used alone, NPWT may increase angiogenesis, but adding 1\% acetic acid soaks enhances re-epithelialization via the upregulation of MMP-1. Through macro- and microdeformation, NPWT also continuously encourages the proliferation of fibroblasts, albeit fibroblast senescence is averted by debridement too [15]. Because chronically infected soft tissue defects are not always amenable to immediate reconstructive surgery, our protocol provides another means to reduce wound size and control infection, allowing time for a patient's general condition to improve.

Admittedly, the findings of this study are preliminary and are based on the few patients studied. However, further research is warranted in this setting, recruiting more patients of this type for similar investigations. NPWT and acetic acid irrigation, when used jointly, help decrease the $\mathrm{pH}$ of chronic wounds, reducing their size and resolving infection so that wound healing may proceed.

\section{ACKNOWLEDGMENTS}

We would like to express our sincere gratitude to Jae Yeon Seok, $\mathrm{MD}, \mathrm{PhD}$, from the Department of Pathology at Gacheon University Gil Medical Center for assistance with the related laboratory studies.

\section{REFERENCES}

1. Arias CA, Murray BE. Antibiotic-resistant bugs in the 21st century: a clinical super-challenge. N Engl J Med 2009;360: 439-43.

2. Merckoll P, Jonassen TO, Vad ME, et al. Bacteria, biofilm and honey: a study of the effects of honey on 'planktonic' and biofilm-embedded chronic wound bacteria. Scand J Infect Dis 2009;41:341-7.

3. de Laat EH, van den Boogaard MH, Spauwen PH, et al. Faster wound healing with topical negative pressure therapy in difficult-to-heal wounds: a prospective randomized controlled trial. Ann Plast Surg 2011;67:626-31.

4. Kim BS, Choi WJ, Baek MK, et al. Limb salvage in severe diabetic foot infection. Foot Ankle Int 2011;32:31-7.

5. Scherer SS, Pietramaggiori G, Mathews JC, et al. The mechanism of action of the vacuum-assisted closure device. Plast Reconstr Surg 2008;122:786-97.

6. Milner SM. Acetic acid to treat Pseudomonas aeruginosa in superficial wounds and burns. Lancet 1992;340:61.

7. Ryssel H, Kloeters O, Germann G, et al. The antimicrobial effect of acetic acid: an alternative to common local antiseptics? Burns 2009;35:695-700.

8. Ryssel H, Gazyakan E, Germann G, et al. Antiseptic therapy with a polylacticacid-acetic acid matrix in burns. Wound Repair Regen 2010;18:439-44.

9. Ryssel H, Germann G, Riedel K, et al. Suprathel-acetic acid matrix versus acticoat and aquacel as an antiseptic dressing: an in vitro study. Ann Plast Surg 2010;65:391-5.

10. Wedam SB, Low JA, Yang SX, et al. Antiangiogenic and antitumor effects of bevacizumab in patients with inflammatory and locally advanced breast cancer. J Clin Oncol 2006;24: 769-77.

11. Gill SE, Parks WC. Metalloproteinases and their inhibitors: regulators of wound healing. Int J Biochem Cell Biol 2008; 40:1334-47.

12. Beidler SK, Douillet CD, Berndt DF, et al. Multiplexed analysis of matrix metalloproteinases in leg ulcer tissue of patients with chronic venous insufficiency before and after compression therapy. Wound Repair Regen 2008;16:642-8.

13. Leonardi A, Cortivo R, Fregona I, et al. Effects of Th2 cytokines on expression of collagen, MMP-1, and TIMP-1 in conjunctival fibroblasts. Invest Ophthalmol Vis Sci 2003;44:183-9.

14. Ladwig GP, Robson MC, Liu R, et al. Ratios of activated matrix metalloproteinase-9 to tissue inhibitor of matrix metalloproteinase-1 in wound fluids are inversely correlated with healing of pressure ulcers. Wound Repair Regen 2002;10: 26-37. 
15. Bosanquet DC, Harding KG. Wound duration and healing rates: cause or effect? Wound Repair Regen 2014;22:143-50.

16. Rippke F, Schreiner V, Schwanitz HJ. The acidic milieu of the horny layer: new findings on the physiology and pathophysiology of skin pH. Am J Clin Dermatol 2002;3:261-72.

17. Leveen HH, Falk G, Borek B, et al. Chemical acidification of wounds. An adjuvant to healing and the unfavorable action of alkalinity and ammonia. Ann Surg 1973;178:745-53.

18. Percival SL, McCarty S, Hunt JA, et al. The effects of $\mathrm{pH}$ on wound healing, biofilms, and antim icrobial efficacy. Wound Repair Regen 2014;22:174-86.

19. Mah TF, O’Toole GA. Mechanisms of biofilm resistance to antimicrobial agents. Trends Microbiol 2001;9:34-9.

20. Kim PJ, Attinger CE, Steinberg JS, et al. Negative-pressure wound therapy with instillation: international consensus guidelines. Plast Reconstr Surg 2013;132:1569-79.

21. Schneider LA, Korber A, Grabbe S, et al. Influence of $\mathrm{pH}$ on wound-healing: a new perspective for wound-therapy? Arch Dermatol Res 2007;298:413-20.

22. Kiyokawa K, Takahashi N, Rikimaru H, et al. New continuous negative-pressure and irrigation treatment for infected wounds and intractable ulcers. Plast Reconstr Surg 2007; 120:1257-65.

23. Ugaki S, Kasahara S, Arai S, et al. Combination of continuous irrigation and vacuum-assisted closure is effective for mediastinitis after cardiac surgery in small children. Interact Cardiovasc Thorac Surg 2010;11:247-51.

24. Greener B, Hughes AA, Bannister NP, et al. Proteases and $\mathrm{pH}$ in chronic wounds. J Wound Care 2005;14:59-61.

25. Leonardi A, Cortivo R, Fregona I, et al. Effects of Th2 cytokines on expression of collagen, MMP-1, and TIMP-1 in conjunctival fibroblasts. Invest Ophthalmol Vis Sci 2003; 44:183-9. 